\title{
Head, Dominic. -- J. M. Coetzee. Cambridge, Cambridge University Press, 1997, 192 p.
}

Jean Sévry

\section{OpenEdition}

1 Journals

Édition électronique

URL : http://journals.openedition.org/etudesafricaines/22

DOI : 10.4000/etudesafricaines.22

ISSN : 1777-5353

Éditeur

Éditions de l'EHESS

\section{Édition imprimée}

Date de publication : 1 janvier 2000

ISBN : 978-2-7132-1346-5

ISSN : 0008-0055

\section{Référence électronique}

Jean Sévry, «Head, Dominic. -- J. M. Coetzee. Cambridge, Cambridge University Press, 1997, 192 p. », Cahiers d'études africaines [En ligne], 157 | 2000, mis en ligne le 24 avril 2003, consulté le 21 septembre 2020. URL : http://journals.openedition.org/etudesafricaines/22 ; DOI : https://doi.org/10.4000/ etudesafricaines.22

\section{Ce document a été généré automatiquement le 21 septembre 2020}

(c) Cahiers d'Études africaines 


\title{
Head, Dominic. -- J. M. Coetzee. Cambridge, Cambridge University Press, 1997, $192 \mathrm{p}$.
}

\author{
Jean Sévry
}

Dominic Head avait publié en 1994, dans la même collection, une étude intéressante sur Nadine Gordimer dont j'avais rendu compte ici ${ }^{1}$. Ce livre constitue donc une sorte de suite dans une série. Or, vouloir maintenant écrire quelque chose sur l'auteur de En attendant les barbares constitue une gageure. En effet, tout critique abordant ce romancier va se heurter nécessairement au moins à cinq écueils. Le premier, c'est qu'il est Afrikaner et Sud-Africain : on peut être alors tenté de tout ramener à ce contexte de production, et à enfermer Coetzee dans ce dilemme, quitte à lui reprocher ses ambiguïtés politiques, un manque d'engagement, ce qui à mon sens, représente une facilité de plus. Le deuxième obstacle, c'est que notre homme a énormément lu, et l'on peut alors s'enliser dans la recherche épuisante d'une interminable intertextualité. Le troisième, c'est qu'il est lui-même au fait des dernières recherches de la critique littéraire, qu'il a un fort savoir linguistique, et l'on aura donc tendance (ce qui s'est produit tout particulièrement pour Foe) à vouloir rechercher à tout prix dans ses romans la trace de ces théories. Le quatrième, c'est qu'il est lui-même un critique de grand talent, ce que l'on retrouve dans White Writing: On the Culture of Letters in South Africa (1988), comme dans Doubling the Point, Essays \& Interviews (1992). En ce cas, on se demandera s'il ne faudrait pas voir dans l'oeuvre l'application de quelque théorie littéraire qui lui tiendrait à coeur. Le cinquième écueil, c'est que l'oeuvre de Coetzee a déjà fait l'objet de nombreuses publications savantes². Les approches varient à l'infini, qu'il s'agisse d'un appel à l'histoire ${ }^{3}$ ou d'un recours à la psychanalyse 4 . Ainsi, peu à peu, se sont mises en place des bienséances universitaires à propos d'un écrivain qui malgré tout n'aura connu qu'une réception limitée pour l'essentiel à son propre milieu, ce qui ne retire rien à ses qualités esthétiques, mais ce qui méritera un jour quelques réflexions sociologiques. OEuvre d'un universitaire composée pour des universitaires? Chacun se sent obligé de faire référence à des concepts tels que " postcolonialism ", "post-modernity», « deconstructivity», « revisionism », et j'en passe, ce qui n'est pas fait 
pour éclaircir le débat, dans la mesure où trop souvent ces vagues concepts sont pris pour argent comptant, politesses obligent. À mon sens, de toute cette littérature, un travail qui est le résultat d'une longue collaboration entre l'auteur et un critique d'une grande finesse, David Atwell (Doubling the Point, 1992) se détache avec beaucoup de grandeur. Head en tient largement compte.

Dans ces conditions, la critique s'interdit généralement une étude transversale de l'oeuvre : ce serait prendre de trop grands risques. On peut le regretter, car c'est une perte de possibilités de rapprochements lesquels seraient enrichissants. Mais cela peut aussi se comprendre dans la mesure où chaque roman de Coetzee finit par constituer une entité d'une densité extrême. Dominic Head ne faillit pas à cette règle, puisque son livre est formé en une introduction suivie de sept chapitres portant sur sept oeuvres prises dans leur ordre chronologique d'apparition, de Dusklands (1974) à The Master of Petersburg (1994). Les soustitres retenus sont fort évocateurs, et chaque chapitre occupe une vingtaine de pages.

Dès les premières pages de son étude ("The Writer's Place »), Head effectue quelques mises en place efficaces, en particulier en ce qui concerne l'identité postcoloniale de Coetzee. Ainsi page 6 (je traduis) : « Coetzee n'est pas un Afrikaner, mais un Blanc sudafricain qui se cantonne dans une marginalité singulière, puisque son milieu le situe quelque peu à l'écart de ses ascendances afrikaner ou anglaises. » De ceci, nous trouvons une étrange confirmation dans la dernière publication de Coetzee, Boyhood, Scenes from a Provincial Life (1997) dont Dominic Head ne pouvait pas nous parler puisqu'il n'était pas encore sorti des presses de Secker \& Warburg. Cette prise de distance lui semble essentielle, car elle justifie également son « scepticisme » à l'égard des modèles occidentaux de narration : la remarque est des plus pertinentes et elle va éviter toute une série de malentendus quant aux « appartenances » de Coetzee. Son indépendance a quelque chose de somptueux, autant que de douloureux.

Par la suite, notre auteur va procéder avec une grande efficacité en se déplaçant d'une oeuvre à l'autre. Je m'arrêterai, à titre d'exemple, au chapitre V, «Gardening as Resistance : "Life and Times of MichaelÊK" ». Head commence par passer en revue les opinions émises à propos de ce chef-d'oeuvre, ainsi par Susan Gallagher qu'il commente à sa façon (page 94) : « En bref, ce roman, dans un style typique de l'ensemble de l'oeuvre, est construit d'une telle façon qu'il procède par allusions à un contexte dont $\mathrm{il}$ se détache au même instant. » Après quoi, il tente de nous montrer comment d'après Derek Wright, Coetzee s'efforce, à travers ce jardinier, de donner le point de vue d'un non-Blanc. Puis on passe à une critique de Nadine Gordimer qui reproche à Coetzee de ne voir en lui que de la passivité au moment, ou cessant de se considérer comme de simples victimes de la ségrégation, les communautés soumises à l'apartheid organisaient leurs luttes. David Atwell insiste sur l'aspect parabolique de l'oeuvre, en termes de pouvoirs. Enfin, Head nous donne son point de vue et fait une lecture attachante de l'oeuvre au travers de Foucault et de ses théories de l'enfermement, à propos de l'épisode crucial du camp de travail forcé de Jakkaldrif (Surveiller et punir, naissance de la prison, 1975). Pour ma part, je crois qu'il eut été intéressant de faire également référence à son Moi, Pierre Rivière, ayant égorgé ma mère, ma soeur et mon frère (1973), en particulier à propos de la figure de l'homme qui ne peut plus que fuir son destin, harcelé par les forces de l'ordre, et qui en est réduit à une survie biologique. Head, avec perspicacité, croit également pouvoir déceler ici un certain retour au réalisme, « un projet de Coetzee qui va de l'avant et qui consiste à ressusciter une forme de réalisme, une sorte de pont plein de vigueur jeté entre le monde et le texte » 
(110). Ainsi il aura consacré huit pages à un panoramique de la critique, ce qui est indispensable, et dix à une expression personnelle. Dans sa recherche de Coetzee en tant qu'écrivain postcolonial, Head a de temps à autre recours à une approche transversale, au travers du personnage de Magda dans Dusklands (1974), de celui du magistrat dans Waiting for the Barbarians (1980), qui représente également la fin du discours libéral. De son côté, dans Age of Iron (1990), Mrs Curren se rend compte qu'elle n'est plus de ce monde : « Elle n'a pas changé d'avis, mais elle a fini par comprendre que ses opinions n'étaient plus de mise » (137).

On regrettera, au passage, que dans le chapitre IV, « An Ethical Awakening: Waiting for the Barbarians ", aucune mention ne soit faite du Désert des Tartares (1945) de Dino Buzzati, dont la trace est pourtant évidente tout au long de En attendant les Barbares. Par contre, le chapitre 3, "The wrong kind of love : in the Heart of the Country », propose des réflexions intéressantes sur l'aspect anti-pastoral de ce roman, et où l'on voit Coetzee prendre ses distances vis-à-vis d'une tradition locale, qu'il s'agisse de Pauline Smith (The little Karoo, 1925) ou d'Olive Schreiner (The Story of an African Farm, 1881, 1899). Son destin de vieille fille (" spinster fate ») évoque pour lui, comme pour Dick Penner qu'il cite encore, l'isolement dans lequel l'Afrique du Sud de l'apartheid se trouvait alors plongée. Pour ce qui est des voix qui traversent le ciel désert de In the Heart of the Country, Dominic Head, à force de ne vouloir entendre que celles d'une situation coloniale moribonde, tend à négliger les références proprement bibliques de ce texte. Il s'agit très nettement, en effet, d'une reprise du Deutéronome (XXVII, 2-4) ${ }^{5}$, ce qui a été entièrement ignoré par la critique. Ceci, à mon sens, lève bien des équivoques : Magda a perdu le sens de la loi mosaïque, elle ne sait plus ce que c'est que la loi, sans doute parce qu'elle a confondu la personne du géniteur avec la figure du Père (ce qui est bien différent), image même de l'autorité. Elle est donc sans parole, sans grammaire, en situation de naufrage ontologique, d'errance éthique, ce qui nous renverrait de ce fait à la chute de l'autorité au sein de la situation coloniale. Un peu plus loin, Head me semble plus proche de cette dimension lorsqu'il nous déclare (69) : "Le célibat de Magda indique que la situation coloniale se réduit à une impasse. » Les conclusions que l'on trouve dans le chapitre VIII, «Producing the Demon: The Master of Petersburg », me semblent fort bien venues. Avec justesse, Dominic Head s'attarde sur le dilemme de l'écrivain sud-africain. Il cite avec raison l'article très important rédigé par Coetzee en 1986 ("Into the Dark Chamber ») dans lequel il dénonçait l'impuissance dans laquelle l'écrivain se retrouve coincé, cette sorte d'obscénité qui fait de lui un voyeur décrivant les horreurs se déroulant dans les chambres obscures de la ségrégation ${ }^{6}$. Il s'agit en effet de savoir de quelle autorité l'auteur peut disposer en tant que voix : elle est des plus minces, puisqu'elle se réduit à un témoignage d'impuissance. Des souvenirs de Dostoïewsky et de Gordimer viennent renforcer ce sentiment ${ }^{7}$. Selon moi, c'est certainement là l'un des sens cachés de l'oeuvre de Coetzee, cette non-pertinence d'une loi qui n'est plus celle du Père, dans une société où la seule brutalité policière prétend la représenter. Les analogies avec la Russie des Tsars sont évidentes, tout autant que les références à l'univers de Kafka. Je m'étonne toujours que l'on ne cite pas (Head non plus) un texte majeur de Kafka qui je pense a profondément marqué Coetzee, $j^{\prime}$ entends "The Cares of a Family Man ${ }^{8}$, où un certain Odradek, sorte de symbolisation de la loi mosaïque, ne parvient plus à donner les repères nécessaires. Il est étonnant de voir à quel point Kafka a pu réveiller des fantasmes et servir de référence vitale au sein de ces écritures blanches sud-africaines. Pour s'en rendre mieux compte, il suffit de se reporter à l'étonnante lettre du père de 
Kafka à Franz, telle qu'elle a été imaginée par Nadine Gordimer dans Something Out There ${ }^{9}$. La critique a trop négligé cet aspect des choses. Car ici, l'histoire devient une dérision, et tout récit est dérisoire.

Mais Dominic Head a malgré tout encore quelques réflexions intéressantes à nous proposer. Ainsi page 160 : «L'écrivain sud-africain qui avec raison ne peut percevoir sa position que comme provisoire, ressent un besoin de mettre en place une autorité reconnue. Dans des termes que l'on pourrait discuter, cette façon de re-centrer le postcolonialisme prend toute sa signification par sa spécificité, par sa précarité. Dans le cas de Coetzee, on peut observer qu'un modèle de décolonisation littéraire qui pourrait n'être jamais qu'une abstraction se met à la recherche d'un lien historique et politique. Cette façon d'examiner la colonisation désigne déjà une destination future, de nouvelles conjonctures, et un nouveau modèle littéraire. »

Les dernières lignes de cette remarquable étude sont écrites dans la même veine (162) : "L'idée même de l'héroïsme est devenue un thème récurrent dans l'oeuvre de Coetzee, et il y a effectivement peut-être quelque chose d'héroïque dans sa façon d'élaborer un projet permettant de créer d'autres nouveaux espaces de l'imaginaire, un versant différent de la créativité. »

\section{NOTES}

1. Cf. Cahiers d'Études africaines, XXXV (4), 140, 1995, pp. 950-954.

2. Consulter en particulier : T. Dovey, The Novels of J. M. Coetzee : Lacanian Allegories, Johannesburg, Ad. Donker \& Craighall, 1998 ; D. Penner, Countries of the Mind : the Fiction of J. M. Coetzee, Westport, Greenwood Press, 1989 ; S. van Zanten Gallagher, A Story of South Africa: J. M. Coetzee's Fiction in Context, Cambridge, Mass, Harvard University Press, 1991 ; M. V. Moses, ed., "The Writings of J. M. Coetzee ", South Atlantic Quaterly, 93, Winter 1994, special issue, Durham, N.C., Duke University Press ; G. Hugan \& S. Watson, eds, Critical Perspectives on J. M. Coetzee, London, Macmillan, 1996. Du côté français : "Waiting for the Barbarians ", Commonwealth, special issue, n SP3, 1992, Université de Dijon ; Les cahiers Forell, $n^{\circ} 3$, 1994, Université de Poitiers. Il faut ajouter à tout ceci de très nombreux articles. Dominic Head, dans sa bibliographie, en recense au moins 102, mais il en existe davantage. Se reporter au recensement effectué sous la direction de T. Dovey en 1990 in J. M. Coetzee. A Bibliography, Grahamstown, NELM Bibliographic Series, $\mathrm{n}^{\circ} 3,103 \mathrm{p}$.

3. S. Gallagher, op. cit.

4. T. Dovey, op. cit.

5. «Et il arrivera que le jour où vous passerez le Jourdain, pour entrer dans le pays de l'Éternel, ton Dieu, te donne, tu te dresseras de grandes pierres, et tu les enduiras de chaux ; et tu écriras sur elles toutes les paroles de cette loi, quand tu auras passé. » Deutéronome, Ancien Testament, version J. N. Darby, Valence, La bonne Semence, 1975, pp. 152-153. 
6. Head redonne également toute son importance à un autre article de Coetzee, "Breyten Breytenbach and the Censor ", in C. Tiffin \& A. Lawson, eds, Describing Empire, Post-Colonialism and Textuality, London, Routhledge \& Kegan Paul, 1994, pp. 86-97.

7. Head évoque alors la scène dans Burger's Daughter de Nadine Gordimer où l'on voit Rosa assistant sans pouvoir intervenir à une scène au cours de laquelle un paysan frappe inutilement un cheval. On retrouve la même scène dans un cauchemar de Raskolnikov dans le Crime et Châtiment de Dostoïevski.

8. F. Kafka, «The Cares of a Family Man », in The Penguin Complete Short Stories of Franz Kafka, London, Penguin Books, 1983, pp. 427-429.

9. N. Gordimer, «Letter from his Father », in Something Out There, Johannesburg, Ravan Press, 1984, pp. 40-56. 